\title{
Ultra-Compact Amorphous Silicon Waveguide for Wavelength Conversion
}

\author{
Cosimo Lacava, Mohamed A. Ettabib, Ilaria Cristiani, Jean Marc Fedeli, David J. Richardson and \\ Periklis Petropoulos.
}

\begin{abstract}
In this work we demonstrate, for the first time, successful four wave mixing (FWM) based wavelength conversion of Binary Phase Shift Keyed (BPSK) and Quadrature Phase Shift Keyed (QPSK) signals, at $20 \mathrm{~Gb} / \mathrm{s}$ bitrate, in a 1-mm long amorphous silicon waveguide. A maximum FWM-efficiency of $-26 \mathbf{d B}$ was achieved by employing a pump power of just $70 \mathrm{~mW}$, establishing this technology as a contender for the development of ultra-compact, low power, silicon photonics wavelength converter. Bit Error Ratio (BER) measurements demonstrated successful conversion with less than $1 \mathrm{~dB}$ penalty level, for both BPSK and QPSK signals (at BER $=10^{-5}$ ).
\end{abstract}

Index Terms-Silicon Photonics, Wavelength Converters, FourWave Mixing, Phase Shift Keying, Optical Waveguide, Integrated Optics, Nonlinear Optics.

\section{INTRODUCTION}

$\mathbf{O}$ VER the last few years Silicon ( $\mathrm{Si}$ ) photonics has been established as a mature technology platform allowing the integration of electronic and optical components in a very compact optoelectronic device. Several important functionalities have already been demonstrated, such as phase and intensity light modulation, as well as on-chip light generation, amplification and detection [1]. In this context, the development of a Si-photonics compatible device for alloptical nonlinear signal processing is a key step towards the achievement of a fully functional on-chip platform for future short- and long-range high speed optical communication networks. In the last few years, a number of alloptical signal processing devices have been demonstrated mainly exploiting cascaded- $\chi^{(2)}$ or $\chi^{(3)}$ nonlinear effects. Thanks to the large $\chi^{(3)}$ coefficient of Si-photonic devices at telecom wavelengths [2], FWM based wavelength converters [3], demultiplexers [3] and high speed all-optical switches [4] implemented in silicon-based waveguides have already been developed. Unfortunately, two-photon absorption (TPA) and TPA-generated free carrier effects (absorption and dispersion, FCA and FCD) must be carefully considered when designing

This work was supported by the Engineering ans Physical Sciences Council (EPSRC) under Grant "Silicon Photonics For Future Systems"; Data can be downloaded from http://dx.doi.org/10.5258/SOTON/381316

Cosimo Lacava, Mohamed A. Ettabib, Dave J. Richardson and Periklis Petropoulos are with the Optoelectronic Research Centre, University of Southampton, SO17 1BJ, Southampton, United Kingdom (email: C.Lacava@soton.ac.uk, mae206@orc.soton.ac.uk, djr@orc.soton.ac.uk, pp@orc.soton.ac.uk)

Ilaria Cristiani is with Dept. of electrical, Computer and Biomedical Engineering, University of Pavia, Via Ferrata 5/A, IT27100, Pavia, Italy (email: ilaria.cristiani@unipv.it)

Jean Marc Fedeli is with CEA Leti Minatec, Campus 17 Rue des Martyrs, 38054, Grenoble, France (email:jean-marc.fedeli@cea.fr) a Si-photonics chip to be operated at telecom wavelengths, as they pose a fundamental limit to the achievable overall efficiency of such devices [1]. In order to overcome this limitation, different approaches have been proposed, including active carrier removal systems [1], slot waveguides [5], and the use of alternative materials [6]. Even though these routes can achieve significant improvements in terms of nonlinear performance, they entail more complex fabrication techniques that could be undesirable for highly integrated systems. Silicon nitride $\left(S i_{3} N_{4}\right)$ waveguide have also been explored in recent years showing very good nonlinear performance [7]. However the relatively low optical confinement that could be achieved in such waveguides strongly reduces the value of the $R e \gamma$ necessitating the use of relatively long waveguides in order to achieve all-optical processing by means of nonlinear effects [7]. Recently amorphous silicon (a-Si) waveguides emerged as a viable alternative to crystalline Silicon (c-Si), showing an increased nonlinear coefficient alongside a reduced TPAfactor [8], [9], [10]. Furthermore, a-Si layers can be grown at temperatures compatible with the back-end line, improving the CMOS material compatibility. These properties offer the potential for the implementation of ultra-compact nonlinear devices, possibly integrated with supporting components, all combined on the same chip. To date however, there have been no investigations on how a-Si waveguides would perform with phase-encoded telecommunication signals. In this paper we present the first successful experimental wavelength conversion of $20 \mathrm{~Gb} / \mathrm{s}$ BPSK and QPSK signals in a mm-long amorphous silicon waveguide, employing just $70 \mathrm{~mW}$ pump power level. This result opens the way to the development of ultra-compact, low power, TPA-free, FWM-based silicon photonics wavelength converters.

\section{Device DeSign And FABrication}

The waveguide cross-section is shown in Fig.1 (inset). The thickness and width are set to $220 \mathrm{~nm}$ and $480 \mathrm{~nm}$ respectively. The sample under test was fabricated starting from a $1.7 \mu \mathrm{m}$ thick $\mathrm{SiO}_{2}$ layer. The hydrogenated a-Si layer was deposited by means of the Plasma Enhanced Chemical Vapour Deposition (PECVD) technique at a temperature of $350{ }^{\circ} \mathrm{C}$. A first etching step was used to define grating coupler patterns with an etching depth of $70 \mathrm{~nm}$. The couplers were designed in such a way to only couple the transverse electric mode (TE-mode). After deposition of a silica hard mask, a full etch step (etching depth of $220 \mathrm{~nm}$ ) was performed to define the waveguide layout. Several straight and serpentine 
waveguides (with a fixed $480 \times 220 \mathrm{~nm}$ cross section) were obtained with lengths varying from $1 \mathrm{~mm}$ to $82.6 \mathrm{~mm}$. Finally, a protective $500 \mathrm{~nm}$ oxide upper cladding was deposited on the top of the waveguides (that will operate as waveguide-cladding layer).

\section{NONLINEAR OPTICAL PROPERTIES AND DISPERSION}

In order to evaluate the nonlinear optical performance of the fabricated waveguides we performed continuous wave $(\mathrm{CW})$ degenerate FWM experiments in the telecom C-band in waveguides of different-lengths. A CW pump signal (@1550.0 nm) was coupled together with a weaker CW signal (@1550.5 nm) by means of a fibre coupler. The resulting optical beam was then sent to the waveguide under test by using a vertical coupling scheme. The propagation losses of the straight waveguide were assessed to be $4.7 \mathrm{~dB} / \mathrm{cm}$, while the coupling losses were estimated to be $10 \mathrm{~dB} /$ grating. We note that this value is significantly higher than expected, probably due to an overetching error occurred in the grating fabrication. By measuring the FWM conversion efficiency (defined as $P_{\text {idler }} / P_{\text {signal }}$ measured at the waveguide output) it was possible to assess the nonlinear parameter $(\operatorname{Re}\{\gamma\})$ by means of the following equation [9]:

$$
\operatorname{Re}\{\gamma\}=\frac{\sqrt{P_{i}(L) / P_{s}(L)}}{\eta P_{p}(0) L_{e f f}}
$$

where $L_{e f f}$ is the effective nonlinear waveguide length, and $\eta$ accounts for the phase mismatch induced by waveguide dispersion. Thanks to the relatively small detuning value between the pump and signal beams the effect of dispersion could be neglected, allowing thus to assume $\eta \approx 1$. By considering spectra obtained from waveguides of different lengths, measured at various pump power levels, we estimated $\operatorname{Re} \gamma=800 \pm 50 \mathrm{~W}^{-1} \mathrm{~m}^{-1}$, which is three times larger with respect to values commonly measured in c-Si waveguides. The waveguide dispersion was also evaluated by measuring the FWM efficiency while increasing the pump - signal detuning, as reported in Fig.1. By fitting the curve, a dispersion coefficient $\mathrm{D} \approx 380 \mathrm{ps} /(\mathrm{nm} \mathrm{km})$ was obtained. In order to the verify the material stability over the time a 6 hours high power injection $(i 200 \mathrm{~mW})$ experiment was carried out observing no degradation for both linear and nonlinear performance.

\section{BPSK AND QPSK CONVERSION EXPERIMENT DESCRIPTION AND RESULTS}

The wavelength conversion experimental set-up is shown in Fig.2. The CW Pump optical beam $(\lambda=1551.3 \mathrm{~nm})$ was generated by means of C-band tuneable laser and amplified by an EDFA; a tap coupler was used to accurately monitor the optical pump power. BPSK $\left(2^{31}-1\right.$ pseudo-random bit sequence (PRBS)) and QPSK (2 $2^{31}-1$ PRBS) optical signals were generated at 20 Gbaud and 10 Gbaud, respectively, starting from a CW C- band signal at $1550.3 \mathrm{~nm}$. Polarization of both pump and signal beams was set to the TE mode by employing fibre polarization controllers. The pump and the modulated Signal were then coupled together by means of a

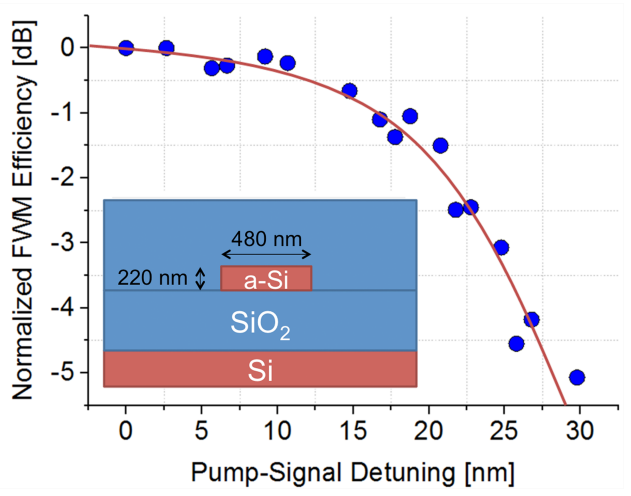

Fig. 1. Normalized FWM efficiency as a function of pump-signal detuning. The waveguide length was $2.26 \mathrm{~cm}$. Inset: Waveguide cross section.

50:50 fibre coupler. Coupling to and from the 1-mm long asilicon waveguide was facilitated through the use of grating couplers that were inscribed at the input and output of the device (10 dB coupling loss per grating). After propagation inside the waveguide the light, that also contained the FWMidler generated signal, was sent to the receiver. In order to remove pump and signal beams a filter allowing both wavelength and bandwidth tuneability was used; the received power level was controlled by using a Variable Optical Attenuator (VOA) and, finally, the light was sent to a real time Optical Modulation Analyzer that allowed us to assess the performance of the a-Si waveguide wavelength converter, both in terms of constellation diagrams and BER measurements.

\section{A. $20 \mathrm{~Gb} / \mathrm{s}$ BPSK Wavelength Conversion}

The first set of experiments was carried out by using a $20 \mathrm{~Gb} / \mathrm{s}$ BPSK modulated signal. The pump power inside the a-Si waveguide (measured taking into account grating coupler losses) was set to $70 \mathrm{~mW}$. CW signal propagation measurements, at this power level, revealed that TPA effects were negligible and that the loss-regime was still purely linear. The optical spectrum recorded at the waveguide output is shown in Fig.3. The measured FWM conversion efficiency was measured to be $-26 \mathrm{~dB}$. Constellation diagrams for both the BSPK signal (back to back configuration) and BPSK idler

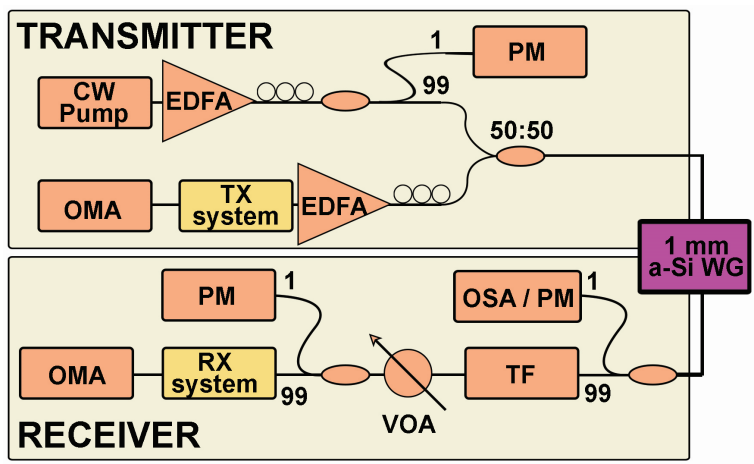

Fig. 2. Wavelength converter experimental apparatus. PM: Power Meter; VOA: Variable Optical Attenuator; OSA: Optical Spectrum Analyser; TF: Tuneable optical Filter; OMA: Optical Modulation Analyzer 


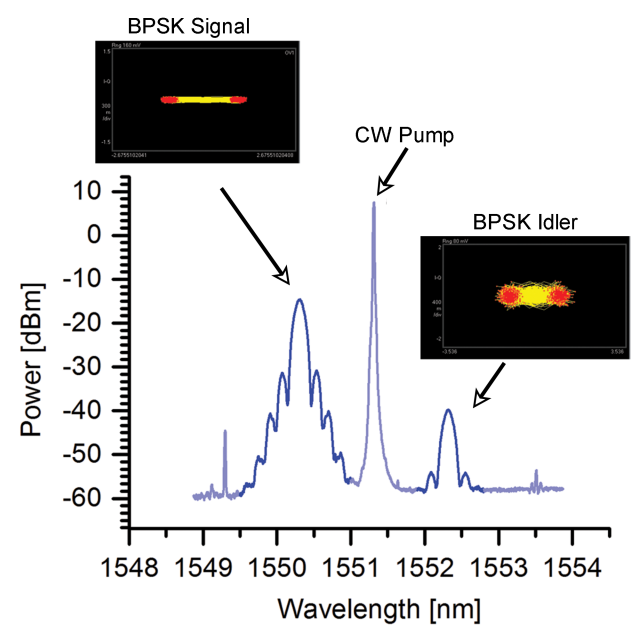

Fig. 3. Output BPSK spectra (resolution $0.05 \mathrm{~nm}$ ) recorded at the waveguide output. Signal and Idler constellation diagrams are also shown as insets

are shown. The rms Error Vector Magnitude (EVM) was also measured by using the Optical Modulation Analyzer, and was equal to $10 \%$ for the signal beam and $22 \%$ for the converted idler, measured after the filtering stage (filter bandwidth set to $1.2 \mathrm{~nm})$. Fig. 4 shows the BER curves measured for the B2B and the converted signal revealing that successful wavelength conversion was obtained with only $1 \mathrm{~dB}$ of power penalty at $\operatorname{BER}=10^{-5}$.

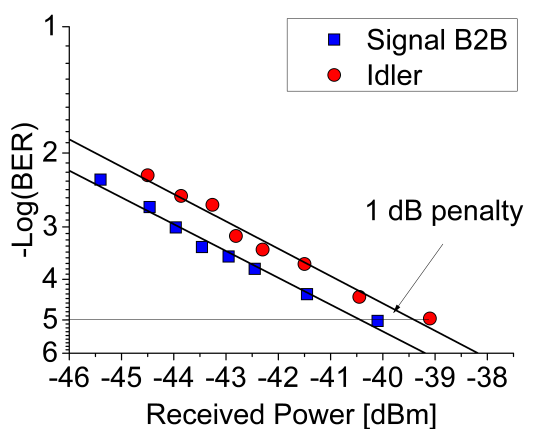

Fig. 4. BER measurements for a $20 \mathrm{~Gb} / \mathrm{s}$ BPSK signal. Input pump power was set to $70 \mathrm{~mW}$.

\section{B. $20 \mathrm{~Gb} / \mathrm{s}$ QPSK Wavelength Conversion}

A second set of experiments was carried out in order to assess the performance of our wavelength conversion system with QPSK signals. Two electrical PRBS $\left(2^{31}-1\right)$ signal were generated by means of an Arbitrary Waveform Generator (AWG) at a rate of 10 Gbaud. The generated waveforms were sent to an I/Q modulator that provided the starting QPSK 20 $\mathrm{Gb} / \mathrm{s}$ signal that was intended to be converted by using the amorphous silicon waveguide. Both signal and pump optical beams were then amplified by means of EDFAs and then coupled together and sent to the a-Si waveguide by using the same experimental scheme shown in Fig.2. Both B2B and idler signal constellation diagrams were recorded by the OMA and are shown in Fig.5 (a) and (b), respectively. The rms EVM was assessed to be $6.45 \%$ for the B2B and $19.748 \%$ in the case of the idler signal. Wavelength converter performance was also assessed by measuring BER as a function of the received power. Results are shown in Fig.5 (c) and reveal that a successful conversion was achieved with a power penalty below $1 \mathrm{~dB}$ at $\mathrm{BER}=10^{-5}$ (note that no BER measurements lower than $10^{-5}$ were possible using the real time acquisition system).

\section{Discussion}

In this section we attempt a brief review and comparison of the performance achieved here with other FWM-based wavelength converting devices realized using different nonlinear and active waveguide platforms. It is also worth comparing this work with these competing technologies by using three key-parameters: i) device length, ii) pump power inside the converter device and iii) obtained FWM efficiency at the output of the device. A normalized efficiency defined as [5]:

$$
\eta_{\text {norm }}=\frac{100 \times\left(P_{\text {idler }} / P_{\text {signal }}\right)}{P_{\text {pump }}^{2} \times L^{2}}
$$

takes into account all these three parameters providing a useful figure of merit for the considered devices. Table I shows the normalized efficiency for four different devices already presented in the literature. Crystalline Silicon waveguides (on a $\mathrm{SiO} 2$ substrate) have been widely used in the past as wavelength converters; as an example in [11] authors show a successful conversion of 28 Gbaud 16 QAM signal using a pump power of $24 \mathrm{dBm}$. Taking into account the length of this device a $0.055 \% \mathrm{~W}^{-2} \mathrm{~mm}^{-2}$ normalized efficiency is obtained showing that this waveguide still requires a relatively high power and relatively large footprint in order to be operated as wavelength converter. Moreover, crystalline silicon suffers from TPA and FCA effects that limit the overall nonlinear efficiency of the final device. In [6] a SiGe waveguide was engineered and operated to act as wavelength converter; QPSK and BPSK signal conversion was successfully obtained with a pump power level of $24.7 \mathrm{dBm}$ in a cm-scale waveguide. Other integrated technologies are also available. In [12] authors showed a Quantum Dot- Semiconductor Optical Amplifier (QD SOA) operated as a wavelength converter with a bias current of 2 A. Even though this device could exhibit comparable efficiencies with respect to Si-based integrated devices, it is not CMOS compatible making the integration more complex. Moreover SOAs are active devices, therefore the fabrication process may include a significantly larger number of steps. Resonant and Photonic Crystal (PhC) devices could also be employed for wavelength conversion purposes. This is the case of the device shown in [13] where authors employed a $\mathrm{GaInP} \mathrm{PhC}$ to convert both a $\mathrm{CW}$ and an OOK signal. The normalized efficiency was assessed to be $23.78 \% \mathrm{~W}^{-2} \mathrm{~mm}^{-2}$. In this case it is worth noting that this efficiency was obtained by operating the device at a frequency very close to the band-edge, therefore the nonlinear performance is strongly dependent on the operating wavelength, dramatically reducing the effective device bandwidth. In contrast to the previous works, in the experiments presented herein we obtained a 


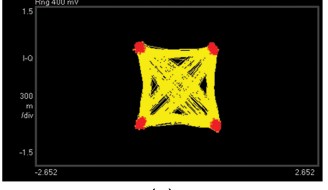

(a)

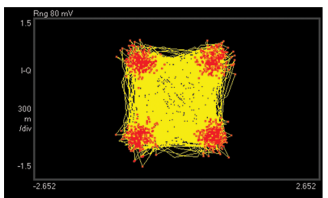

(b)

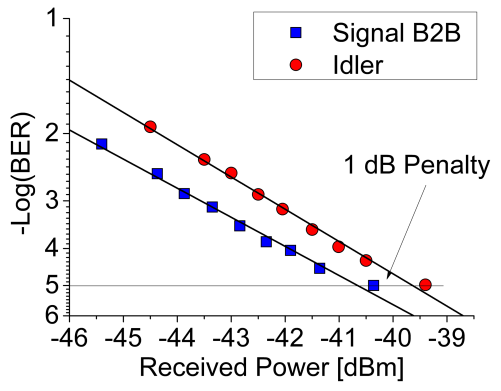

(c)

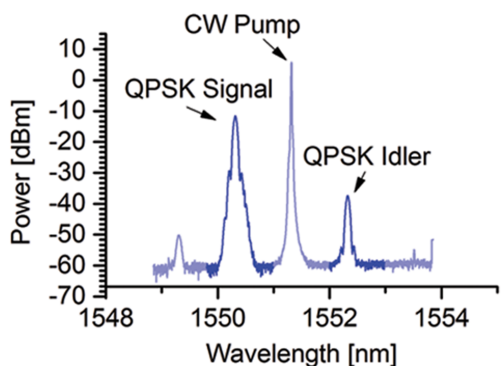

(d)

Fig. 5. $20 \mathrm{~Gb} / \mathrm{s}$ QPSK constellation diagram (a) signal and (b) idler. (c) QPSK BER measurements (d) recorded spectra for QPSK wavelength conversion experiment

normalized efficiency of $51.26 \% \mathrm{~W}^{-2} \mathrm{~mm}^{-2}$ which is to the best of our knowledge the highest value reported in literature to date, for a integrated wavelength converter, and highlights the potential of this technology.

\section{CONCLUSION}

In this work we demonstrated FWM-based conversion of a $20 \mathrm{~Gb} / \mathrm{s}$ BPSK and QPSK signals in an ultra-short $(1 \mathrm{~mm})$ a-Si waveguide. Thanks to the enhanced nonlinear response with respect to its c-Si counterparts, this waveguide could be operated at moderate pump power levels, with no evidence of TPA-related effects. In order to evaluate the conversion capabilities of our device we employed both constellation diagrams and BER testing, demonstrating a conversion with only a $1 \mathrm{~dB}$ power-penalty at $\mathrm{BER}=10^{-5}$ for both tested signals. It is worth noting that this result was obtained by using only a $1-\mathrm{mm}$ waveguide and a pump power level of $70 \mathrm{~mW}$. Therefore, this represents a first very promising step towards the achievement of compact, low power FWM -based converters for complex telecommunication signals. Our results are compromised mainly by the poor coupling efficiency to the waveguide and secondly by the propagation losses. Improvements upon these two factors would allow using both higher pump power levels and longer waveguides that could lead to a significant improvement in FWM-efficiency. By considering the measured nonlinear parameter of our waveguides we estimated that we could approach the 0-dB FWM efficiency level by using a $L_{e f f}=10$-mm waveguide operated at a pump power of $120 \mathrm{~mW}$ which is still well below the TPA power limit.

\section{REFERENCES}

[1] V. Almeida, C. Barrios, R. Panepucci, and M. Lipson, "All-optical control of light on a silicon chip," Nature, vol. 431, pp. 1081-1084, 2004.

[2] M. Dinu, F. Quochi, and H. Garcia, "Third-order nonlinearities in silicon at telecom wavelengths," Applied Physics Letters, vol. 82, no. 2003, pp. 2954-2956, 2003.

[3] C. Koos, P. Vorreau, T. Vallaitis, and P. Dumon, "All-optical high-speed signal processing with silicon organic hybrid slot waveguides," Nature photonics, vol. 3, no. April, pp. 1-4, 2009.

[4] C. Lacava, M. J. Strain, P. Minzioni, I. Cristiani, and M. Sorel, "Integrated nonlinear Mach Zehnder for $40 \mathrm{Gbit} / \mathrm{s}$ all-optical switching," Optics Express, vol. 21, no. 18, p. 21587, Sep. 2013.
TABLE I

DIFFERENT PLATFORM FWM-BASED WAVELENGTH CONVERTERS

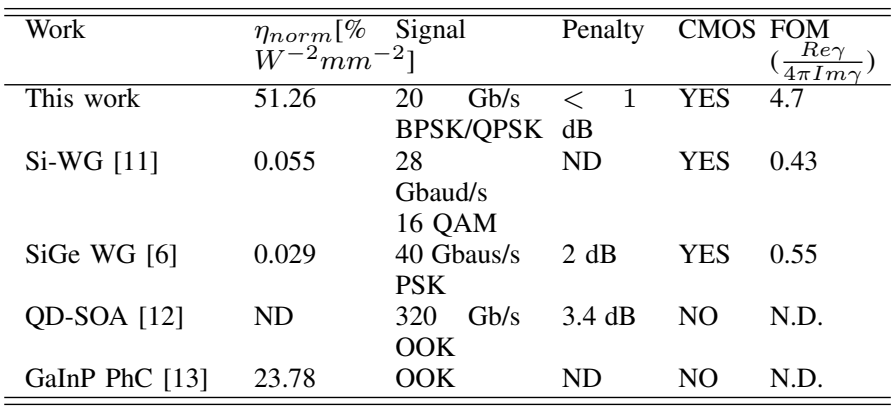

[5] A. Trita, C. Lacava, P. Minzioni, J. Colonna, P. Gautier, J. Fedeli, and I. Cristiani, "Ultra-high four wave mixing efficiency in slot waveguides with silicon nanocrystals," Applied Physics Letters, vol. 99, no. 19, p. 191105,2011

[6] M. a. Ettabib, K. Hammani, F. Parmigiani, L. Jones, A. Kapsalis, A. Bogris, D. Syvridis, M. Brun, P. Labeye, S. Nicoletti, and P. Petropoulos, "FWM-based wavelength conversion of 40 Gbaud PSK signals in a silicon germanium waveguide," Optics Express, vol. 21, no. 14, pp. 1723, 2013.

[7] Y. Okawachi, K. Saha, J. S. Levy, Y. H. Wen, M. Lipson, and A. L. Gaeta, "Octave-spanning frequency comb generation in a silicon nitride chip." Optics letters, vol. 36, no. 17, pp. 3398-3400, 2011.

[8] B. Kuyken, H. Ji, S. Clemmen, S. Selvaraja, H. Hu, M. Pu, M. Galili, P. Jeppesen, G. Morthier, S. Massar, and Others, "Nonlinear properties of and nonlinear processing in hydrogenated amorphous silicon waveguides," Optics express, vol. 19, no. 26, pp. B146-B153, Dec. 2011.

[9] C. Lacava, P. Minzioni, E. Baldini, L. Tartara, J. M. Fedeli, and I. Cristiani, "Nonlinear characterization of hydrogenated amorphous silicon waveguides and analysis of carrier dynamics," Applied Physics Letters, vol. 103, no. 141103, p. 4, 2013.

[10] K.-Y. Wang and A. C. Foster, "Ultralow power continuous-wave frequency conversion in hydrogenated amorphous silicon waveguides," Optics letters, vol. 37, no. 8, pp. 1331-1333, Apr. 2012.

[11] R. Adams, M. Spasojevic, M. Chagnon, M. Malekiha, J. Li, D. V. Plant, and L. R. Chen, "Wavelength conversion of 28 GBaud 16-QAM signals based on four-wave mixing in a silicon nanowire." Optics express, vol. 22, no. 4, pp. 4083-90, 2014

[12] M. Matsuura, O. Raz, F. Gomez-Agis, N. Calabretta, and H. J. S. Dorren, "320 Gbit/s wavelength conversion using four-wave mixing in quantumdot semiconductor optical amplifiers." Optics letters, vol. 36, no. 15, pp. 2910-2912, 2011.

[13] V. Eckhouse, I. Cestier, G. Eisenstein, S. Combrié, P. Colman, A. De Rossi, M. Santagiustina, C. G. Someda, and G. Vadalà, "Highly efficient four wave mixing in GaInP photonic crystal waveguides." Optics letters, vol. 35, no. 9, pp. 1440-1442, May 2010 . 